\title{
Epigenetic Aberrant Hypermethylation of DNA in Endometrial Cancer: Application as a Biomarker
}

\author{
Asuka Ono, Iori Kisu, Kouji Banno*, Megumi Yanokura, Kenta Masuda, Yusuke Kobayashi, \\ Kosuke Tsuji, Arisa Ueki, Wataru Yamagami, Hiroyuki Nomura, Nobuyuki Susumu, Daisuke Aoki
}

Department of Obstetrics and Gynecology, School of Medicine, Keio University, Tokyo, Japan.

E-mail: *kbanno@sc.itc.keio.ac.jp

Received October $15^{\text {th }}$, 2011; revised November 20 ${ }^{\text {th }}, 2011$; accepted November 30 ${ }^{\text {th }}, 2011$.

\begin{abstract}
Endometrial cancer is the seventh most common cancer worldwide among females and accounts for about $40 \%$ of cancers of the uterus in Japan. An increase in incidence and a reduction in onset age of this disease are also likely, which makes it important to define the pathogenesis and develop effective treatment. However, the mechanism of canceration in the endometrium is unclear and development of endometrial cancer cannot be explained only by mutations of cancer-related genes. In contrast, epigenetic analyses have shown the importance of aberrant DNA hypermethylation in the canceration mechanism. In development of type 1 endometrial cancer, breakdown of the DNA mismatch repair system plays a large role, with changes in the human mutL homologue 1 ( $h M L H 1)$ gene being of most importance. Studies to detect aberrant DNA hypermethylation of cancer cells present in microscopic amounts in vivo and to apply these data to diagnosis of cancer have been started. Epigenetic changes have also been examined as a marker of sensitivity to anticancer drugs. Aberrant hypermethylation of checkpoint with forkhead-associated and ring finger (CHFR), a mitotic phase checkpoint gene, is correlated with sensitivity to treatment with microtubule inhibitors and may be a marker for the response of endometrial cancer to anticancer drugs. Epigenetic aberrant DNA methylation of other genes may also be useful as clinical biomarkers for diagnosis and treatment of endometrial cancer.
\end{abstract}

Keywords: Epigenetics, Endometrial Cancer, Aberrant DNA Hypermethylation, hMLH1, CHFR, Biomarker

\section{Introduction}

Epigenetics refers to information other than the DNA base sequence that is directly stored at the time of somatic cell division. It has become apparent that certain aberrant genetic functions do not depend on DNA sequence mutations, but on epigenetic changes, and that these changes are involved in carcinogenic mechanisms in various organs. Associations between cancer and aberrant hypermethylation in specific genome regions are of particular interest. These findings have led to a new model of carcinogenesis in which a CpG island in a cancer-related gene promoter that is not methylated in normal cells becomes hypermethylated, with resultant inactivation of the gene (so-called silencing) and induction of canceration. Many cancer-related genes with aberrant methylation have been reported, including $p 16, A P C$, and human mutL homologue 1 ( $h M L H 1$ ), in multiple types of cancers [1,2], indicating the complexity of the processes of canceration, genetic changes, and epigenetic alteration of genes. A major difference between genetic changes, such as gene mutations, and epigenetic changes, which do not involve a change in base sequence, is the reversible nature of epigenetic alterations. Thus, re-expression of an epigenetically silenced gene may be possible through the action of a suitable molecule; that is, epigenetic aberrations can be a molecular target for treatment. In this context, trials have been initiated to detect DNA aberrant methylation in cancer cells present in microscopic amounts in vivo and to apply the findings as biomarkers for diagnosis and classification of cancer and the risk of canceration.

\section{Epigenetic Aberrant DNA Hypermethylation}

Epigenetic mechanisms of regulation of gene expression include DNA methylation, histone modification, and effects of polycomb group proteins [3]. DNA methylation is imprinted in the genome at the time of cell division and has been widely studied as a representative epigenetic modification in mammals. In DNA methylation 
in vertebrates, methyl groups are added to cytosine bases at CpG sites. This modification is performed by DNA methyltransferase enzymes, including Dnmt1, Dnmt2, Dnmt3a, Dnmt3b, and Dnmt3L. These enzymes are divided into those associated with maintenance of methylation and those that produce new methylation. DNA methylation in CpG islands in promoters upstream of the start of gene transcription has an important role in gene expression [4]. When the DNA in this region is methylated, nucleosomes impede transcription estricting access of transcription factors to their target DNA sequences [5, 6] (Figure 1). Many anticancer genes, such as $C D K N 2 A$, $C D H 1$ (E-cadherin) and $h M L H 1$, are inactivated by DNA methylation in promoter CpG islands, which suggests that aberrant DNA methylation is involved in carcinogenesis [7].

\section{Epigenetic Aberrant DNA Hypermethylation in Endometrial Cancer}

Cases of endometrial cancer are defined as type 1 or 2 based on clinicopathological properties. Type 1 endometrial cancer often develops in premenopausal or perimenopausal women and occurs in an estrogen-dependent manner via hyperplasia. The tumor is positive for the estrogen receptor and the progesterone receptor, shows G1 or G2 well-differentiated endometrioid adenocarcinoma morphologically, has a lower frequency of lymph node metastasis, shows little muscle invasion, and often has a relatively favorable prognosis. In contrast, type 2 endometrial cancer tends to develop in postmenopausal women in an estrogen-independent manner, and is thought to develop directly from the normal endometrium, rather than via hyperplasia or from undiagnosed precancerous lesions. The tumors are endometrioid adenocarcinoma or serous adenocarcinoma with extremely low differentiation, and often have a poor prognosis.

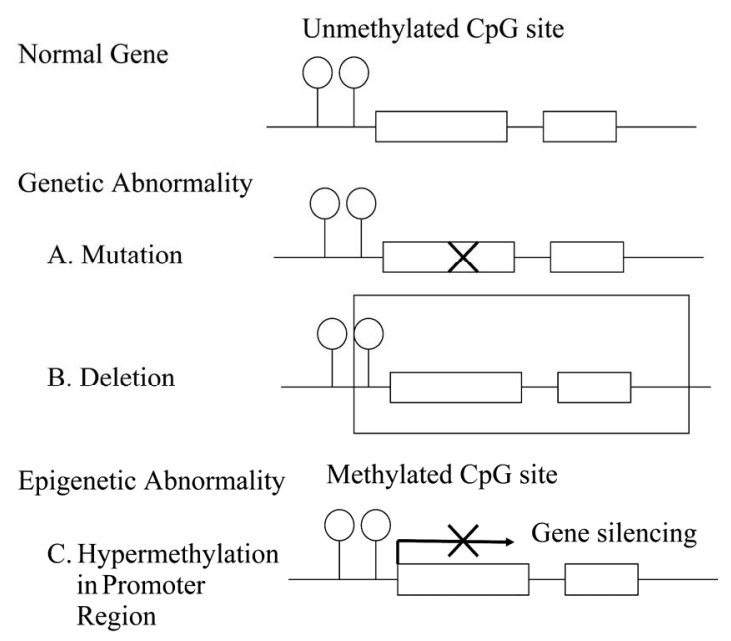

Figure 1. Inactivation mechanism of cancer-related genes.
An epigenetic mechanism has been proposed for development of type 1 endometrial cancer, with the epigenetic aberration causing a breakdown of the DNA mismatch repairing system. This system corrects nucleotide mismatches occurring during DNA replication. Inactivation of DNA mismatch repair genes leads to an accumulation of mutations that eventually causes canceration. $h M L H 1$ is the main mismatch repair gene that is inactivated by DNA methylation in endometrial cancer, with $40 \%$ of cases showing this epigenetic change [8]. Inactivation of $h M L H 1$ is an important step in the early stage of canceration of the uterus. In endometrial cancer, methylation of $h M L H 1$ causes loss of DNA mismatch repair functions and induces mutation of PTEN and other genes [9].

Microsatellite instability (MSI) is a characteristic of tumor cells in which the mismatch repair system has failed. Microsatellites in genes are repeat sequences of about 1 - 5 bases that are particularly prone to replication mistakes in the absence of mismatch repair, with a resultant change in the number of repeats in the sequence. This change is referred to as microsatellite instability and is found in certain types of cancer. It is especially prevalent in endometrial cancer, in which $20 \%$ - 30\% of cases show MSI [10]. These results suggest that changes in MMR genes occur frequently in endometrial cancer.

We detected aberrant methylation of $h M L H 1$ in $40.4 \%$ of cases of endometrial cancer, and similar aberrant methylation of $A P C, E$-cadherin, and checkpoint with forkhead-associated and ring finger (CHFR) in 22.0\%, 14.0\%, and $13.3 \%$ of cases, respectively [8]. In cases with aberrant methylation of $h M L H 1$ and E-cadherin, protein expression was significantly reduced (hMLH1: p $<0.01$, E-cadherin: p < 0.05). Aberrant methylation of $h M L H 1$ was also detected in $14.3 \%$ of cases of atypical endometrial hyperplasia (AEH). However, in the normal endometrium, none of the four cancer-related genes were aberrantly methylated. These results suggest that aberrant methylation of specific genes (and particularly $h M L H 1$ ) is important in carcinogenesis of endometrial cancer, particularly since methylation of $h M L H 1$ was found in $\mathrm{AEH}$, which is viewed as the early stage of type 1 endometrial cancer [8] (Figure 2).

\section{Application of Aberrant DNA Hypermethylation to Diagnosis}

Analysis of aberrant methylation in microscopic amounts of DNA is possible at high sensitivity using the PCR method. This information may permit diagnosis and targeting of cancer. Methylated genes from cancer cells can be detected using methylation-specific PCR (MSP), in which bisulfite treatment and PCR are combined. Cytosine is converted to uracil by bisulfite treatment, but me- 
thylated cytosine is not changed by this reaction. MSP is then performed using PCR primers that detect sequences containing cytosine or uracil, allowing the absence or presence of methylation to be detected through PCR amplification. This method can be used to detect aberrant methylation in sputum, plasma, and urine samples. In endometrial cancer, detection of aberrant methylation of particular genes has been examined using specimens collected for intimal cytology (Figure 3).

In addition to $h M L H 1$, several other genes have been reported to be methylated in endometrial cancer. These include CASP8, an apoptosis-related gene [11]; TGF$\beta R I I$, which codes for a receptor of $T G F-\beta$, which is involved in signaling that inhibits cell proliferation [12]; $p 73$, a cancer suppressor [11], HOXA11, which is important in the development of the uterus [13], and COMT, the gene for catechol O-methyltransferase, a metabolizing enzyme for catechol estrogen, which is a carcinogen [14]. Methylation of each of these genes reduces protein expression and promotes canceration, since the degree of methylation differs between normal and endometrial cancer tissue [15].

Aberrant DNA methylation can also influence the properties of cancer cells. In colorectal cancer, methylation of CHFR is detected in $40 \%$ of cases and is associated with the sensitivity of tumor cells to microtubule inhibitors such as docetaxel (taxotere) [16]. Methylation of $M G M T$, a DNA repair enzyme, alters the sensitivity to alkylating agents [17], simultaneous methylation of $C D K N 2 A$ and FHIT is associated with recurrence of lung cancer [18], and methylation of $E R-\alpha$ has been linked to prognosis after tamoxifen treatment [19].

\begin{tabular}{|c|c|c|c|c|c|c|}
\cline { 2 - 7 } \multicolumn{1}{c|}{} & hMLIII & L-cadherin & APC & CIIIR & RAR- $\beta$ & pl6 \\
\hline \multirow{2}{*}{$\mathrm{EC}$} & $40.4 \%$ & $14.0 \%$ & $22.0 \%$ & $12.8 \%$ & $2.3 \%$ & $0 \%$ \\
& $(21 / 52)$ & $(7 / 50)$ & $(11 / 50)$ & $(6 / 47)$ & $(1 / 44)$ & $(0 / 44)$ \\
\hline \multirow{2}{*}{$\mathrm{AEH}$} & $14.3 \%$ & $0 \%$ & $7.3 \%$ & $0 \%$ & $0 \%$ & $0 \%$ \\
& $(2 / 14)$ & $(0 / 14)$ & $(1 / 14)$ & $(0 / 14)$ & $(0 / 10)$ & $(0 / 8)$ \\
\hline \multirow{2}{*}{$\mathrm{NE}$} & $0 \%$ & $0 \%$ & $0 \%$ & $0 \%$ & $0 \%$ & $0 \%$ \\
& $(0 / 18)$ & $(0 / 20)$ & $(0 / 18)$ & $(0 / 10)$ & $(0 / 10)$ & $(0 / 7)$ \\
\hline
\end{tabular}

EC: Endometrial cancer

AEH: Atypical endometrial hyperplasia

NE: Normal endometrium

Upper row: Frequency

Lower row: Number of cases

Figure 2. Frequency of aberrant hypermethylation in each gene in endometrial cancer.

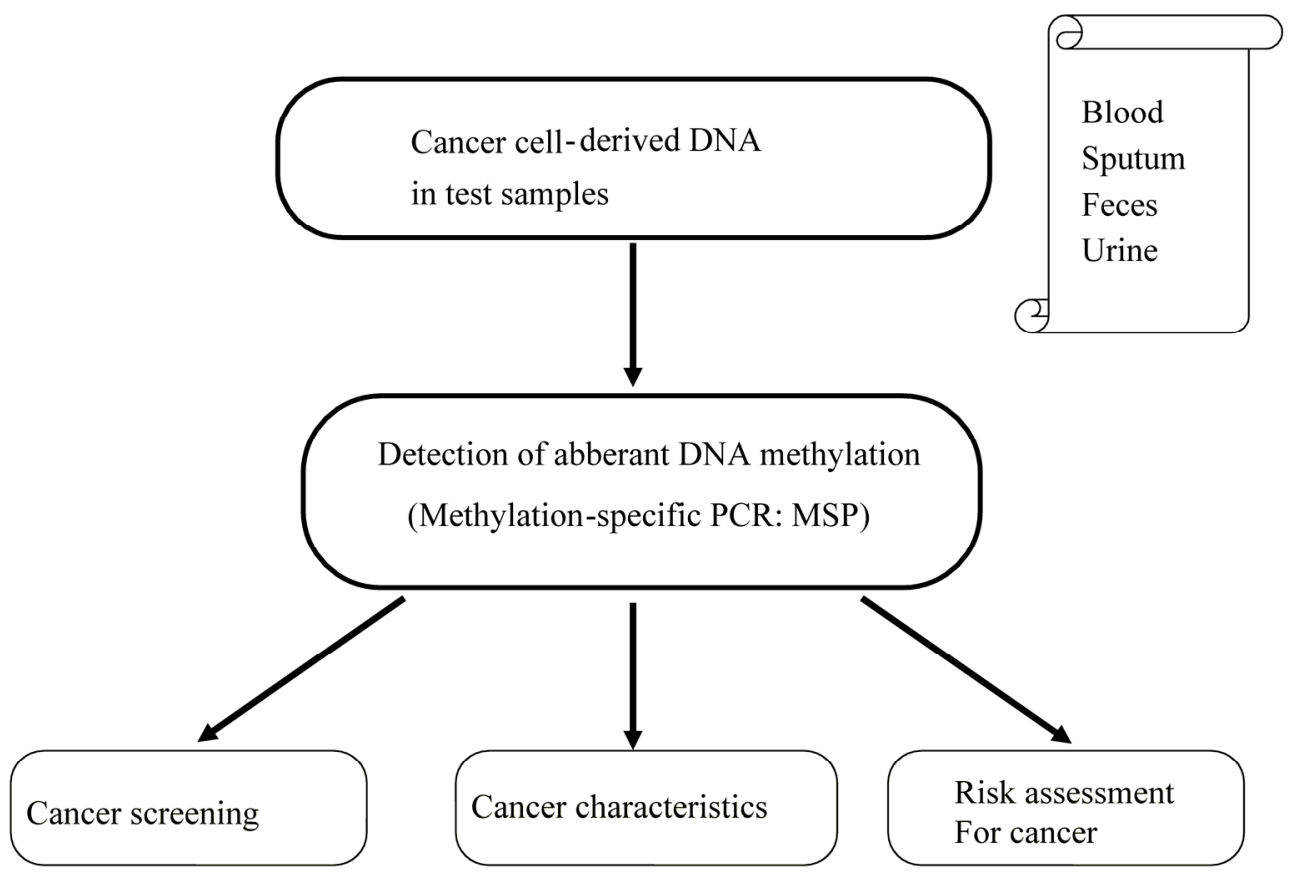

Figure 3. Diagnostic applications of abberant DNA hypermethylation in cancer. 
Cadherins are a family of cell-cell adhesion milecules essential for tight connection between cells. E-cadhein is the major cadherin molecule that keeps neighboring cell attached. Impaired function of this molecule can lead to differentiation of the tumor cells and increase in metastatic potential [20]. Metylation on the E-cadherin was frequently found in poorly-differentiated endometrial cancer (G3). Metylation of the E-cadherin has been associated with tumor differentiation, myometrial invasion and prognosis in endometrial cancer [20,21].

\section{Application of Aberrant DNA Hypermethylation to Treatment}

Use of agents to inhibit DNA methylation is an old anticancer treatment, with trials of these agents for treatment of leukemia being performed in the 1960s. However, toxicity caused by administration of a high dose of these drugs was problematic and prevented practical use. More recently, antitumor effects have been obtained at lower doses with fewer side effects, and better efficacy may be achievable through combination with other chemotherapeutic agents. Lubbert et al. obtained a response rate of $60 \%$ using 5-aza-dC, a methylation inhibitor, in patients with myelodysplastic syndrome [22]. In cases that were responsive to 5-aza-dC, expression of $p 15 I N K 4 A$ was detected following demethylation. Issa et al. also obtained a response rate of $60 \%$ with repeated administration of a low dose of 5-aza-dC in cases of acute myelocytic leukemia [23]. However, a problem with this approach is that demethylation is not sequence specific and removal of methyl groups with biological importance or activation of oncogenes that are silenced by methylation may occur. Therefore, agents are being developed that can, for example, demethylate the DNA sequence bound by a particular transcription factor.

Utilization of epigenetic aberrations as markers of sensitivity to anticancer drugs is also being examined. Satou et al. found that aberrant methylation of CHFR, a $\mathrm{M}$ phase checkpoint gene, is correlated with the sensitivity of tumors to microtubule inhibitors [24] (Figures 4 and 5). This finding suggests that treatment may be selected based on the specific methylation properties of tumor cells. Methylation of CHFR also plays an important role in development of endometrial cancer and therefore may also be a marker of sensitivity of endometrial cancer to anticancer drugs. CHFR includes a fork-head associated (FHA) domain and a RING finger domain, and was first identified as a homolog of the yeast $D M A 1$ gene [25]. If mitotic stress occurs during $\mathrm{M}$ phase, $C H F R$ delays the progress from early prophase to late prophase. $C H F R$ is also an ubiquitin ligase with substrates including Aurora$\mathrm{A}$ and PLK1. If microtubule stress occurs, CHFR is activated, decomposes Aurora-A and PLK1, and arrests the cell cycle [26,27]. The FHA domain may be associated with phosphorylated proteins and is important for checkpoint control, but further analysis is required to establish the molecules that interact with $C H F R$.

CHFR is methylated at high rates in cancer $[16,26]$ and this has been proposed to have the following effect on the mitotic index. In normally functioning cells without detectable methylation of $C H F R$, cell division is stopped in the G2/M phases by administration of docetaxel and the mitotic index is low. In contrast, cells in which expression of $C H F R$ is reduced by methylation, a high mitotic index is found after administration of docetaxel; thus, the expression level of $C H F R$ and the mitotic index are inversely correlated [16]. In some cells, the checkpoint mechanism of action in response to mitotic stress involves transition of cyclin B1 to the nucleus, which is unaffected by $C H F R$ methylation. In addition, if cells in which CHFR is methylated are treated with 5aza-dC, the checkpoint mechanism is restored and the mitotic index is reduced. Methylation of $C H F R$ is a useful

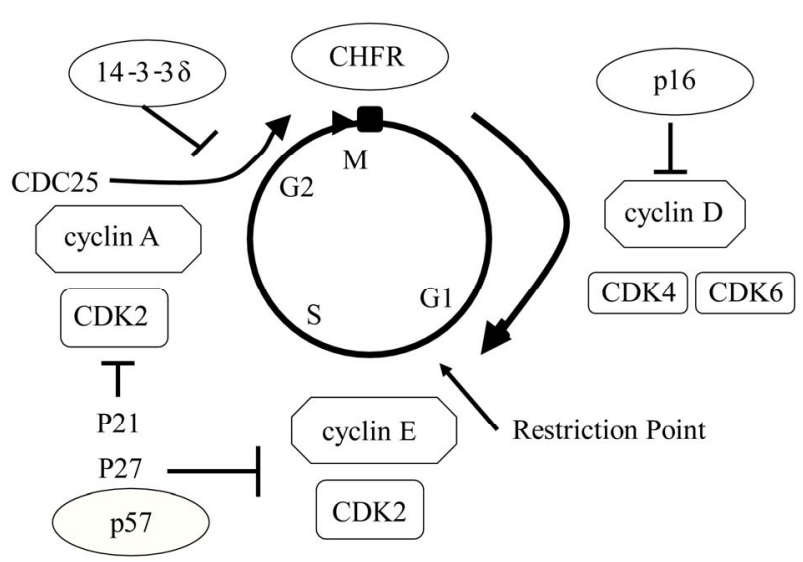

Figure 4. CHFR (checkpoint with forkhead-associated and ring finger) as cell cycle checkpoint genes.

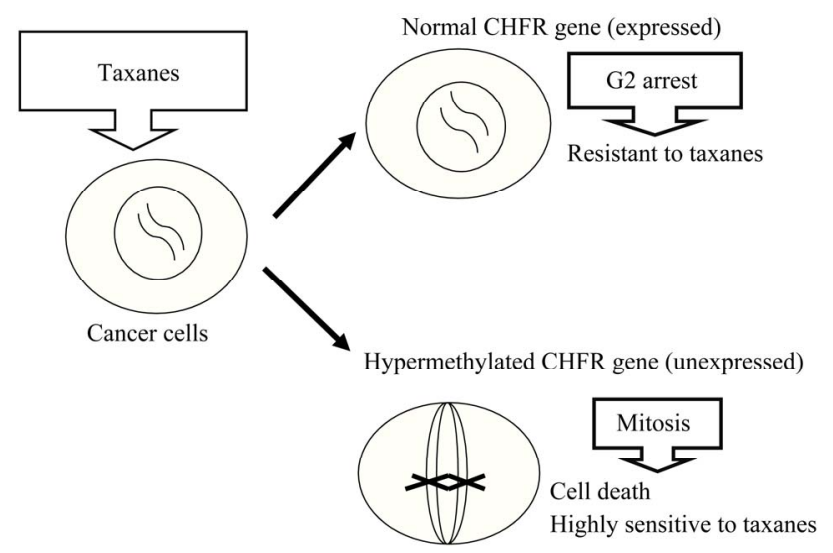

Figure 5. Relationship between expression of CHFR and sensitivity of cancer cells to taxanes. 
measure of sensitivity to microtubule inhibitors in endometrial cancer and in stomach and cervical cancer, and the methylation index may become a useful marker for prediction of the effects of anticancer drugs [28-31].

\section{Conclusions}

Progress in epigenetics has shown that aberrant DNA methylation is correlated with the developmental mechanisms of several kinds of cancers, including endometrial cancer. In type 1 endometrial cancer, breakdown of DNA mismatch repair due to epigenetic silencing of $h M L H 1$ is an important factor. These kind of epigenetic data are likely to lead to discovery of biomarkers for prevention, diagnosis, risk evaluation, and treatment of endometrial cancer, with considerable potential for clinical application. Diagnostic applications based on epigenetic changes could include methylation of genes specific to particular cancers using detection methods such as MSP and samples from bone marrow, peripheral blood, and biopsy materials. Studies toward this goal have been performed for several cancers, including endometrial cancer. Currently, the main goals of epigenetic studies in cancer research are to identify gene hypermethylation that is directly related to canceration and to utilize these findings in diagnosis and treatment. Agents that regulate methylation of specific genes may ultimately be particularly effective as anticancer therapy.

\section{REFERENCES}

[1] S. B. Baylin and J. G. Herman, "DNA Hypermethylation in Tumorigenesis: Epigenetics Joins Genetics,” Trends in Genetics, Vol. 16, No. 4, 2000, pp. 168-174. doi:10.1016/S0168-9525(99)01971-X

[2] M. Esteller, P. G. Corn, S. B. Baylin and J. G. Herman, "A Gene Hypermethylation Profile of Human Cancer," Cancer Research, Vol. 61, No. 8, 2001, pp. 3225-3229.

[3] A. D. Goldberg, C. D. Allis and E. Bernstein, "Epigenetics: A Landscape Takes Shape,” Cell, Vol. 128, No. 4, 2007, pp. 635-638. doi:10.1016/j.cell.2007.02.006

[4] T. Ushijima, "Detection and Interpretation of Altered Methylation Patterns in Cancer Cells," Nature Reviews Cancer, Vol. 5, No. 3, 2005, pp. 223-231. doi:10.1038/nrc1571

[5] E. N. Gal-Yam, S. Jeong, A. Tanay, G. Egger, A. S. Lee and P. A. Jones, "Constitutive Nucleosome Depletion and Ordered Factor Assembly at the GRP78 Promoter Revealed by Single Molecule Footprinting," PLoS Genetics, Vol. 2, No. 9, 2006, p. 160. doi:10.1371/journal.pgen.0020160

[6] R. Appanah, D. R. Dickerson, P. Goyal, M. Groudine and M. C. Lorincz, “An Unmethylated 3'Promoter-Proximal Region Is Required for Efficient Transcription Initiation,” PLoS Genetics, Vol. 3, No. 2, 2007, p. 27. doi:10.1371/journal.pgen.0030027
[7] P. A. Jones and S. B. Baylin, "The Epigenomics of Cancer,” Cell, Vol. 128, No. 4, 2007, pp. 683-692. doi:10.1016/j.cell.2007.01.029

[8] K. Banno, M. Yanokura, N. Susumu, M. Kawaguchi, N. Hirao, A. Hirasawa, K. Tsukazaki and D. Aoki, "Relationship of Aberrant DNA Hypermethylation Of CancerRelated Genes with Carcinogenesis of Endometrial Cancer,” Oncology Reports, Vol. 16, No. 6, 2006, pp. 11891196.

[9] M. Kawaguchi, K. Banno, M. Yanokura, Y. Kobayashi, A. Kishimi, S. Ogawa, I. Kisu, H. Nomura, A. Hirasawa, N. Susumu and D. Aoki, “Analysis of Candidate Target Genes for Mononucleotide Repeat Mutation in Microsatellite Instability-High (MSH-H) Endometrial Cancer," International Journal of Oncology, Vol. 35, No. 5, 2009, pp. 977-982.

[10] T. Kanaya, S. Kyo, Y. Maida, N. Yatabe, M. Tanaka, M. Nakamura and M. Inoue, "Frequent Hypermethylation of MLH1 Promoter in Normal Endometrium of Patients with Endometrial Cancers,” Oncogene, Vol. 22, No. 15, 2003, pp. 2352-2360. doi:10.1038/sj.onc.1206365

[11] H. J. Yang, V. W. Liu, Y. Wang, P. C. Tsang and H. Y. Ngan, "Differential DNA Methylation Profiles in Gynecological Cancers and Correlation with Clinico-Pathological Data,” BMC Cancer, Vol. 6, 2006, p. 212. doi:10.1186/1471-2407-6-212

[12] J. Sakaguchi, S. Kyo, T. Kanaya, Y. Maida, M. Hashimoto, M. Nakamura, K. Yamada and M. Inoue, “Aberrant Expression and Mutations of TGF-Beta Receptor Type II Gene in Endometrial Cancer," Gynecologic Oncology, Vol. 98, No. 3, 2005, pp. 427-433. doi:10.1016/j.ygyno.2005.04.031

[13] B. P. Whitcomb, D. G. Mutch, T. J. Herzog, J. S. Rader, R. K. Gibb and P. J. Goodfellow, "Frequent HOXA11 and THBS2 Promoter Methylation, and a Methylator Phenotype in Endometrial Adenocarcinoma," Clinical Cancer Research, Vol. 9, No. 6, 2003, pp. 2277-2287.

[14] M. Sasaki, M. Kaneuchi, N. Sakuragi and R. Dahiya, "Multiple Promoters of Catechol-O-Methyltransferase Gene Are Selectively Inactivated by CpG Hypermethylation in Endometrial Cancer," Cancer Research, Vol. 63, No. 14, 2003, pp. 3101-3106.

[15] H. Fiegl, C. Gattringer, A. Widschwendter, A. Schneitter, A. Ramoni, D. Sarlay, I. Gaugg, G. Goebel, et al., "Methylated DNA Collected by Tampons: A New Tool to Detect Endometrial Cancer," Cancer Epidemiology, Biomarkers \& Prevention, Vol. 13, No. 5, 2004, pp. 882-888.

[16] M. Toyota, Y. Sasaki, A. Satoh, et al., "Epigenetic Inactivation of CHFR in Human Tumors," Proceedings of the National Academy of Sciences, Vol. 100, No. 13, 2003, pp. 7818-7823. doi:10.1073/pnas.1337066100

[17] M. Esteller and J. G. Herman, “Generating Mutations but Providing Chemosensitivity: The Role of O6-Methylguanine DNA Methyltransferase in Human Cancer," Oncogene, Vol. 23, No. 1, 2004, pp. 1-8.

[18] J. S. Kim, J. W. Kim, J. Han, Y. M. Shim, J. Park and D. H. Kim, " Cohypermethylation of p16 and FHIT Promot- 
ers as a Prognostic Factor of Recurrence in Surgically Resected Stage I Non-Small Cell Lung Cancer," Cancer Research, Vol. 66, No. 8, 2006, pp. 4049-4054. doi:10.1158/0008-5472.CAN-05-3813

[19] T. Mori, S. R. Martinez, S. J. O’Day, et al., “Estrogen Receptor-Alpha Methylation Predicts Melanoma Progression,” Cancer Research, Vol. 66, No. 13, 2006, pp. 66926698. doi:10.1158/0008-5472.CAN-06-0801

[20] T. Saito, M. Nishimura, H. Yamasaki and R. Kudo, "Hypermethylation in Promoter Region of E-cadherin Gene Is Associated with Tumor Differentiation and Myometrial Invasion in Endometrial Cancer," Cancer, Vol. 97, No. 4, 2003, pp. 1002-1009. doi:10.1002/cncr.11157

[21] T. Z. Yi, J. Guo, L. Zhou, X. Chen, R. R. Mi, Q. X. Qu, J. H. Zheng and L. Zhai, " Prognotic Value of E-cadherin Expression and CDH1 Promoter Hypermethylation in Patients with Endometrial Cancer," Cancer Investigation, Vol. 29, No. 1, 2011, pp. 86-92. doi:10.3109/07357907.2010.512603

[22] M. Daskalakis, T. T. Nguyen, C. Nguyen, et al., "Demethylation of a Hypermethylated P15/INK4B Gene in Patients with Myelodysplastic Syndrome by 5-Aza-2'-Deoxycytidine [Decitabine] Treatment," Blood, Vol. 100, No. 8, 2002, pp. 2957-2964. doi:10.1182/blood.V100.8.2957

[23] J. P. Issa, G. Garcia-Manero, F. J. Giles, et al., "Phase 1 Study of Low-Dose Prolonged Exposure Schedules of the Hypomethylating Agent 5-aza-2'-Deoxycytidine [Decitabine] in Hematopoietic Malignancies,” Blood, Vol. 103, No. 5, 2003, pp. 1635-1640. doi:10.1182/blood-2003-03-0687

[24] A. Satoh, M. Toyota, F. Itoh, et al., "Epigenetic Inactivation of CHFR and Sensitivity to Microtubule Inhibitors in Gastric Cancer,” Cancer Research, Vol. 63, No. 24, 2003, pp. 8606-8613.

[25] D. M. Scolnick and T. D. Halazonetis, "CHFR Defines a Mitotic Stress Checkpoint That Delays Entry into Metaphase,” Nature, Vol. 406, No. 6794, 2000, pp. 430-435. doi:10.1038/35019108

[26] D. Kang, J. Chen, J. Wong and G. Fang, “The Checkpoint Protein CHFR Is a Ligase That Ubiquitinates Plk1 and Inhibits Cdc2 at the G2 to M Transition,” Journal of Cell Biology, Vol. 156, No. 2, 2002, pp. 249-259. doi:10.1083/jcb.200108016

[27] X. Yu, K. Minter-Dykhouse, L. Malureanu, et al., "CHFR Is Required for Tumor Suppression and Aurora A Regulation,” Nature Genetics, Vol. 37, No. 4, 2005, pp. 401406. doi:10.1038/ng1538

[28] K. Ogi, M. Toyota, H. Mita, et al., "Small Interfering RNA-Induced CHFR Silencing Sensitizes Oral Squamous Cell Cancer Cells to Microtubule Inhibitors," Cancer Biology \& Therapy, Vol. 4, No. 7, 2005, pp. 773-780. doi:10.4161/cbt.4.7.1896

[29] K. Banno, M. Yanokura, M. Kawaguchi, et al., "Epigenetic Inactivation of the CHFR Gene in Cervical Cancer Contributes to Sensitivity to Taxanes," International Journal of Oncology, Vol. 31, No. 4, 2007, pp. 713-720.

[30] Y. Koga, Y. Kitajima, A. Miyoshi, K. Sato, S. Sato and K. Miyazaki, "The Significance of Aberrant CHFR Methylation for Clinical Response to Microtubule Inhibitors in Gastric Cancer,” Journal of Gastroenterology, Vol. 41, No. 2, 2006, pp. 133-139. doi:10.1007/s00535-005-1732-7

[31] Y. Muraki, K. Banno, M. Yanokura, et al., "Epigenetic DNA Hypermethylation: Clinical Applications in Endometrial Cancer (Review)," Oncology Reports, Vol. 22, No. 5, 2009, pp. 967-972. 